\title{
Regards
}

\section{Jean-Marie Legay, les conditions de l'indiscipline}

\author{
Claude Millier \\ Biomathématicien, président de NSS-Dialogues, 92000 Nanterre, France
}

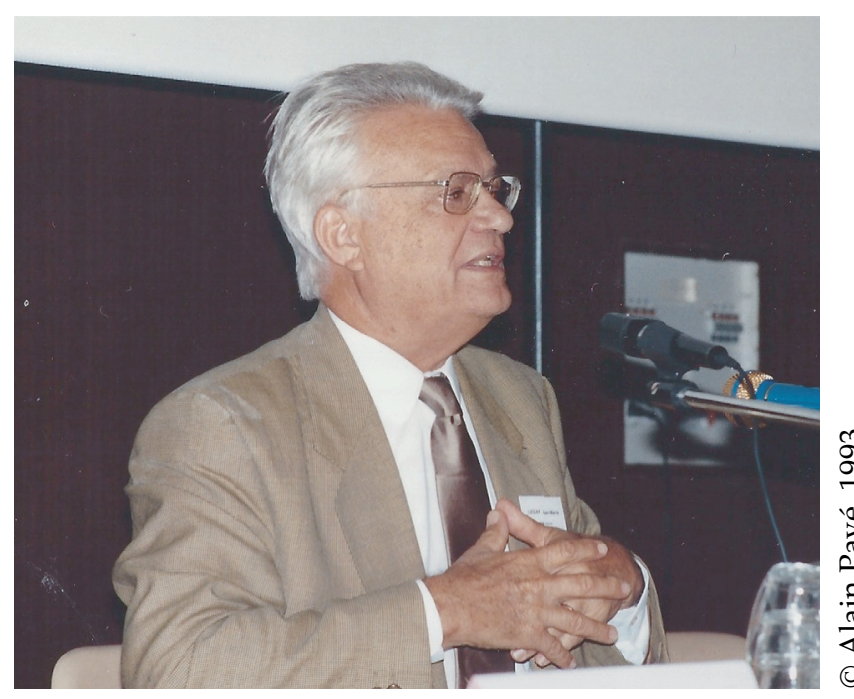

Onze contributeurs ont nourri notre hommage à JeanMarie Legay ${ }^{1}$ : qu'ils en soient remerciés ; de leurs témoignages aux formes et tonalités différentes se dégagent évidemment des convergences sur l'homme, sur son œuvre et sur son influence mais ces témoins, collègues de l'aventure NSS ou étudiants de Legay, sont tous partis de leur relation effective avec lui : il ne faut donc pas s'étonner de l'absence de références aux décennies 1950 et 1960, trop anciennes et pourtant déterminantes pour expliquer beaucoup de traits saillants de sa personnalité.

Bien sûr, ce sont ses années de jeunesse, bases évidentes dans la construction de tout homme, mais ce sont

Auteur correspondant : claude.millier@agroparistech.fr

1 Voir, dans ce numéro, les textes d'Anne-Françoise Schmid, Nicole Mathieu, Sylvie Lardon, Dominique Pontier et Alain Pavé, et, dans le précédent, ceux de Marcel Jollivet, JeanDominique Lebreton, Franck Varenne, Nigel Yoccoz, Richard Tomassone et Francis Laloë. aussi des années de fureur quand la bascule politique s'opère du programme du Conseil national de la Résistance vers la mise en ordre atlantique dans l'atmosphère de la guerre froide. Pour Jean-Marie Legay, ces années sont celles de l'éviction rapide de Georges Teissier de la direction du CNRS, celles de l'impossibilité de concilier liberté du chercheur et pratique au sein de l'Inra, ce qui le conduit à l'Université - refuge ô combien précieux en raison de son statut plus autonome -, celles de son engagement international, depuis la lutte contre le maccarthysme (voir, par exemple, dans l'hommage de Franck Varenne $^{2}$, l'importance de l'influence de Rashevsky, accueilli à Lyon par Legay) jusqu'à ses prises de responsabilités au sein de la Fédération mondiale des travailleurs scientifiques (FMTS). Tout cela mériterait une analyse plus approfondie que nous espérons poursuivre, au sein de NSS ou ailleurs, car ces premières expériences innervent toutes les qualités décrites par les onze contributeurs dans leurs hommages.

J'ai eu l'occasion de travailler avec Jean-Marie Legay, depuis le début des années 1980, dans le comité « Gestion des ressources naturelles renouvelables » de la Délégation générale à la recherche scientifique et technique - où avec Charles Sauvage et Hervé Bichat (et beaucoup d'autres qu'on retrouvera ultérieurement dans l'émergence de NSS), il a contribué à renouveler les thématiques de la gestion des ressources naturelles dans une ambiance interdisciplinaire - jusqu'aux années 19801990, lors du développement d'une politique de thèses en modélisation, en particulier pour des étudiantsingénieurs.

Les nouveaux besoins de la recherche agronomique avaient exigé le recrutement de chercheurs spécialisés appelés à l'époque (1965) «biométriciens ». J'avais été

\footnotetext{
2 Varenne, F., 2012. Quelques aspects de l'œuvre de JeanMarie Legay, NSS, 20, 4, 461-463.
} 
engagé à l'Inra dans cette optique, à un moment où le modèle de la Grande-Bretagne (à l'instar des centres de recherche de Rothampstead, Merlewood, Hurley) avait été pris pour développer la rigueur et l'efficacité dans l'expérimentation agronomique et biologique et pour s'appuyer sur les mathématiques en vue d'accompagner la dialectique théorie-observations (ce qui sera plus tard chez Legay la triade théorie-modélisation-expérimentation $^{3}$ ) ; l'organisation choisie mêlait intimement recherche et "service» aux agronomes et biologistes, compte tenu qu'à cette période les moyens de calculs statistiques étaient obligatoirement concentrés.

La situation a progressivement évolué avec une diffusion de l'utilisation des mathématiques dans le processus de recherche en biologie et une appropriation des moyens de calcul par les chercheurs : à l'Inra, les recrutements des chercheurs et ingénieurs en biométrie évoluant vers des profils de plus en plus (trop ?) spécialisés, les évaluations des carrières privilégiant les publications dans des revues disciplinaires mathématiques, l'organisation administrative de l'Institut faisant du département de biométrie un département comme les autres ${ }^{4}$ ont conduit à des situations caricaturales ; comme la fameuse histoire du rabbin hassidique d'un shtetl, qui courait les rues le soir du shabbat en criant «J'ai une réponse, qui a une question ? ", il y avait un éloignement de plus en plus marqué entre l'activité du biométricien très disciplinaire et l'expression de la demande des biologistes ; cette crise a donc conduit à une autonomie du département de biométrie avec la définition d'objectifs propres.

Pour Jean-Marie Legay, cette situation était incompréhensible, vue de son laboratoire à Lyon; il ne se reconnaissait pas dans les orientations du département de biométrie de l'Inra et il était d'ailleurs plus engagé auprès des chercheurs du département $\mathrm{SAD}^{5}$ du même Inra, dès sa création : le travail sur la formulation de la question est nécessaire et non trivial ; il doit être mené en partenariat entre le biologiste et le biométricien et il induit les caractéristiques du problème «mathématique » à résoudre ; ce travail, difficile à rentabiliser dans une organisation disciplinaire de la production de la recherche, a donc été réduit globalement au sein du département de

3 Legay, J.-M., 1973. La méthode des modèles, état actuel de la méthode expérimentale, Informatique et Biosphère, 1, 5-73.

4 L'extraordinaire débat d'idées qui avait eu lieu en 1968 à l'Inra avait conduit à faire une différence entre les objets de recherche des disciplines classiques, étudiés au sein des départements de recherche, et les objets de recherche des disciplines «transversales »-comme la biométrie et la microbiologie constitués en "commissions spécialisées", beaucoup plus ouvertes à d'autres disciplines ; ces propositions n'ont pas tenu face à la volonté « égalitaire » de tout harmoniser au sein d'une organisation en départements.

5 «Systèmes agraires et développement », à l'époque. biométrie de l'Inra, ce qui a conduit les modèles Inra et lyonnais à s'éloigner progressivement. J'ai été, dans ce débat, hélas assez peu public, très proche des options de Jean-Marie Legay6.

La construction progressive, tenace et efficace de ce modèle, toujours dans une tension évidente, porte la marque de Legay : elle a été sûrement le premier laboratoire de ses pratiques interdisciplinaires (voir aussi les exemples donnés par Franck Varenne ${ }^{7}$ ). Dans la mise en œuvre de ce modèle, la formation doctorale des étudiants était un bon exemple d'application, stratégique quant à ses possibilités de dissémination : ce travail avec JeanMarie Legay, Alain Pavé, Jean-Dominique Lebreton, Daniel Cheyssel - et d'autres - a été pour moi un laboratoire personnel crucial, débouchant sur la nécessité de marier excellence scientifique et pertinence, mon chemin personnel vers NSS.

Certes, en prenant cet exemple des rapports mathématiques/biologie, on voit bien l'apport de Legay dans la mise en place d'une interdisciplinarité entre ces deux disciplines; mais on doit alors déplacer la question en se demandant comment on peut devenir un indiscipliné de qualité, comme aimait à se définir Jean-Marie Legay ${ }^{8}$.

Je proposerais trois qualités différentes et nécessaires, que j'ai entrevues et appréciées chez Legay, qui rendent convaincante une indiscipline efficace et créatrice :

- Une vision cohérente et globale du monde : on est ici très loin d'une simple curiosité sur les sciences et les conditions de leur production, d'une incorporation minimaliste d'idées, de concepts ou de méthodologies dans son champ de réflexion. La vision globale légitime l'indiscipline qui se construit au fil de l'évolution des concepts et des expériences personnelles ; sans elle, l'indiscipline est trop peu fondée et ne peut résister correctement à la critique des idées et à la mise en question des pratiques ; Jean-Marie Legay a été amené à se confronter à des problèmes apparemment relativement éloignés de son cœur d'action. Par exemple, le cours de Legay sur l'asymétrie du vivant restera pour moi l'illustration la plus rayonnante de cette indiscipline bâtie sur une vision globale.

- Une maîtrise de la dialectique théorie/pratique : en travail discipliné, cette maîtrise est une qualité fondamentale qui s'exprime au sein d'un corpus théorique bien délimité et qui demeure implicite, tellement elle est au cœur de l'activité ; en travail indiscipliné, les allersretours entre la construction théorique et les faits sont

6 On trouvera dans la contribution de Richard Tomassone une position un peu différente ! $C f$., dans les hommages du précédent numéro, Tomassone, R., 2012. Jean-Marie Legay, du bon usage de la biométrie, NSS, 20, 4, 466-468.

7 Varenne, F., op. cit.

8 D'ailleurs, à son initiative, en complément de la revue NSS, l'association NSS-Dialogues a créé une collection d'ouvrages intitulée « Indisciplines ». 
plus complexes, en raison du grand nombre de points de vue et d'actions possibles; cette approche doit être obligatoirement explicitée et elle est au centre des débats ; Jean-Marie Legay possédait au plus haut point cette pratique.

- Un mode de traitement du doute : si le doute reste le standard chez les chercheurs et, en particulier, chez Legay, il prend une couleur particulière en travail interdisciplinaire : il y est beaucoup plus complexe et multidimensionnel que dans le travail disciplinaire; il s'agit donc d'abord d'ordonner ce doute correctement; selon moi, la pratique de Jean-Marie Legay était basée sur un traitement à deux niveaux : un premier niveau très personnel, peu communicable, débouchait sur des constructions déjà assez achevées qui, dans un deuxième temps, pouvaient être discutées plus collectivement ; la rigueur de Legay dans les échanges avec ses partenaires (parfois presque décriée) peut être vue à travers cette pratique !

Bien sûr, il existe sûrement d'autres voies vers l'indiscipline mais la trajectoire de Jean-Marie Legay restera pour beaucoup de ses collègues et de ses étudiants un modèle... difficile à atteindre ! 